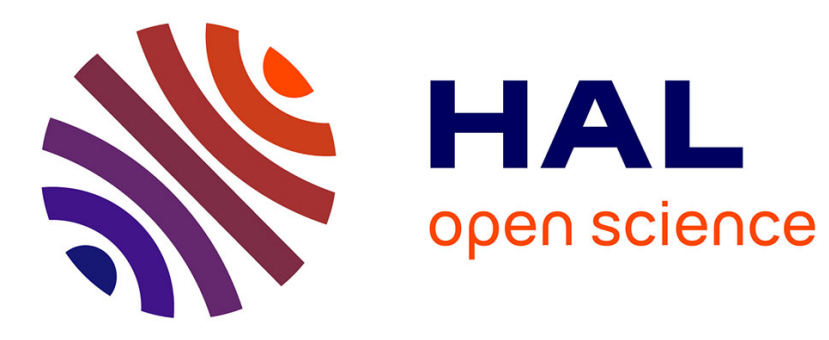

\title{
Experience with the Mobile4D Disaster Reporting and Alerting System in Lao PDR
}

\author{
Ahmed Loai Ali, Jasper van De Ven, Thatheva Saphangthong, Christian \\ Freksa, Thomas Barkowsky, Sithong Thongmanivong, Houngphet \\ Chanthavong, Peter Haddawy
}

\section{To cite this version:}

Ahmed Loai Ali, Jasper van De Ven, Thatheva Saphangthong, Christian Freksa, Thomas Barkowsky, et al.. Experience with the Mobile4D Disaster Reporting and Alerting System in Lao PDR. 14th International Conference on Social Implications of Computers in Developing Countries (ICT4D), May 2017, Yogyakarta, Indonesia. pp.525-535, 10.1007/978-3-319-59111-7_43 . hal-01650086

\section{HAL Id: hal-01650086 \\ https://hal.inria.fr/hal-01650086}

Submitted on 28 Nov 2017

HAL is a multi-disciplinary open access archive for the deposit and dissemination of scientific research documents, whether they are published or not. The documents may come from teaching and research institutions in France or abroad, or from public or private research centers.
L'archive ouverte pluridisciplinaire HAL, est destinée au dépôt et à la diffusion de documents scientifiques de niveau recherche, publiés ou non, émanant des établissements d'enseignement et de recherche français ou étrangers, des laboratoires publics ou privés. 


\title{
Experience with the Mobile4D Disaster Reporting and Alerting System in Lao PDR
}

\author{
Ahmed Loai Ali ${ }^{1}$, Jasper van de Ven ${ }^{1}$, Thatheva Saphangthong ${ }^{2}$, Christian \\ Freksa $^{1}$, Thomas Barkowsky ${ }^{1}$, Sithong Thongmanivong ${ }^{3}$, Houngphet \\ Chanthavong $^{3}$, and Peter Haddawy ${ }^{4}$ \\ 1 [loai, jasper, barkowsky, freksa] @capacitylab.org, \\ Capacity Lab, Bremen Spatial Cognition Center, University of Bremen \\ 2 thatheva@cst-maf.la, Lao Ministry of Agriculture and Forestry, \\ 3 [sithong, houngphet] @nuol.edu.la, \\ Faculty of Forestry, National University of Laos, \\ 4 peter.had@mahidol.ac.th, Faculty of ICT, Mahidol University
}

\begin{abstract}
Information and Communication Technology (ICT) is used to support developing countries in many different ways, such as poverty reduction, public services enhancement, and disaster management and recovery. Mobile4D is a software framework that applies the crowdsourcing paradigm to facilitate information exchange between people during disaster situations. It acts as a disaster reporting and alerting system as well as an information sharing platform. Mobile4D facilitates rapid communication between local citizens and administrative units. Moreover, it allows exchanging experience and knowledge between people to reduce poverty and increase living standards. The Mobile4D framework has been deployed in a pilot study in three provinces in the Lao People's Democratic Republic (Lao PDR). The study was limited to report particular types of disasters, however, it revealed further use cases and identified the required extension of Mobile4D to cover the entire country. This paper presents a report about Mobile4D: initiative, challenges, status, and further extensions.
\end{abstract}

Keywords: ICT4D, crowdsourcing, volunteered geographic information, disaster management, location based services, mobile technologies

\section{Introduction}

Evolution of Information and Communication Technology (ICT) played a major role in various fields like disaster management and poverty reduction in developing countries [11,19]. In particular, ubiquity of Internet and broadband communication channels empowers ordinary people to voluntarily contribute information. With the vast availability of location-sensing devices, advances in Web 2.0, and GeoWeb technologies people are enabled to contribute Volunteered Geographic Information (VGI), which has a significant role in disaster relief $[9,10]$. Natural and human-made disasters usually result in destruction and in the worst case 
in deaths. Although there is no possibility to eliminate the risk of a disaster, projects utilizing ICT demonstrated that the damage caused by disasters can be reduced by proactive planning, mitigation, and rapid response [19]. For example, Ushahidi $^{5}$ and Sahana ${ }^{6}$ are the most common software frameworks that employ ICT to support disaster management activities $[2,18]$. Their functionalities focus mainly on post-activities of disaster management; they support decision makers and aids organizations to coordinate actions and to allocate resources during disaster relief. In contrast, this paper presents Mobile4D software framework that concerned with pre-activities of disaster management. Mobile4D provides location-based early stage reporting and alerting functionalities.

This paper shows research-in-progress of Mobile4D. The paper comprises the initiative, the development status, the pilot deployment in parts of the Lao People's Democratic Republic (Lao PDR), the encountered challenges, and the future plans. The system has been developed in the Capacity Lab ${ }^{7}$ at the University of Bremen, Germany. The Capacity Lab was established to support development and to achieve poverty reduction in developing countries though applying advanced ICT. Lao PDR is a landlocked country located in Southeast Asia, bordered by Myanmar, China, Vietnam, Cambodia, and Thailand. About $80 \%$ of Lao's population are involved in agriculture activities. Such activities are directly influenced by natural disasters like floods, drought, and epidemic diseases. Therefore, Mobile4D is built upon the potential role of ICT to support such essential activities.

Mobile4D is a software framework that facilitates rapid communication between Lao district officers and administrative units in disaster situations. In case of disaster, such grass-root communication is required to provide insights into the real situation. The framework employs mobile technology to achieve broader and rapid utilization. Since 2015, Mobile4D is in use in three Lao provinces collecting early stage reports about disasters.

Development and deployment of Mobile4D encountered various challenges related to technical and non-technical issues. For instance, the system is mostly used in the early stage of disasters, when the situation requires rapid communication and easy-of-use. The reports should be delivered to users in rural and urban areas as well. Based on location, alerts should also be sent to the expected hazard locations. Moreover, the issued reports by non-authorities should be verified by a certain way to ensure their occurrences. Limited experiences of users might results in imprecise use of the system. Therefore, the development team considered all these challenges to develop a reliable system.

The remainder of the paper is organized as follows: Related works are discussed in Section 2; The initiative of Mobile4D is presented in Section 3; Section 4 demonstrates Mobile4D use cases; A technical overview of the system is given in Section 5; The experience and lessons learned are presented in Section 6; whereas Section 7 discusses future work and further development of the system.

\footnotetext{
${ }^{5}$ https://www .ushahidi.com/

${ }^{6}$ https://sahanafoundation.org/

7 http://capacitylab.org/
} 


\section{Related work}

Since the end of 1990s, the Internet and the Millennium Development Goals (MDGs) particularly inspired the evolution of ICT for development (ICT4D), when governmental and non-governmental organizations (NGOs) harnessed the ICT to achieve more development in various domains: education, agriculture, employment, health, ...etc.. Several projects were successful executed to support poverty reduction, such as, e-choupal ${ }^{8}$ and Katha ${ }^{9}$ in India and the Expanded Public Works Programme ${ }^{10}$ (EPWP) in South Africa. Whereas other projects encountered sustainability or scalability problems [1], such as, the Economic Transformation Programme ${ }^{11}$ (ETP) in the Malaysian government [16].

The ubiquity of the Internet, wireless communication technologies, and location aware devices moved ICT4D to another era $[12,13]$. In this era, ICT empowered public citizens to collect and exchange information following the crowdsourcing paradigm. After the Haiti earthquake on 2010, the potential role of crowdsourcing to support disaster relief had been recognized [8]. Several crowdsourcing platforms were used to report about this catastrophic event $[15,17]$. Although social media and crowdsourcing can be utilized for reporting and information propagation in disaster situations, they do not support early warning capabilities or easily coordination of responses. Therefore, dedicated software platforms have been developed - utilizing advance of ICT - to support disaster management activities. So far, most of these platforms focuses on responses coordination and resources allocation (post-activities) in disaster situations, while limited ones consider early stage warning and alerting (pre-activities) toward proactive disaster management plans.

In the last decade, increase availability of Mobile devices fostered mobilebased ICT applications in various domains. The authors in [4] review various mobile-based health applications, while the research in [14] proposes SMS based disaster alert system in developing countries. Moreover, web and mobile applications of voluntary-based flood risk management approach are presented in [5]. Although utilizing mobile technologies allows implementation of low cost systems, development and deployment of these systems encounter various challenges particularly in developing countries; Low communication broadband, limited experience of users, and ensuring the data quality are among other challenges that encounter mobile-based ICT applications in developing countries.

Most of previous research focus on collaboration and coordination of rescue activities, while Mobile4D supports location-based early warning and alerting functionalities. Various applications have been developed addressing a particular disaster in a certain location (e.g., district), while Mobile4D aims to support various kinds of disasters and targets national coverage. It takes into account the information propagation and integration among different levels of decision makers.

\footnotetext{
${ }^{8}$ http://www . itcportal.com/businesses/agri-business/e-choupal . aspx

${ }^{9}$ http://www.katha.org/

10 http://www.epwp.gov.za/

11 http://etp.pemandu.gov.my/
} 


\section{The History of Mobile4D}

In 2007, the Lao Ministry of Agriculture and Forestry (MAF) embarked on a program to strengthen the capacity of staff at the local level as part of the country's poverty reduction strategy. In collaboration with the Wetlands Alliance ${ }^{12}$, the Ministry piloted an innovative professional Bachelors degree program in Poverty Reduction and Agriculture Management (PRAM) ${ }^{13}$ to provide broad skills at the grass-root level. In contrast to the piecemeal short-term training that many development projects provide, the PRAM program provided students with a more complete spectrum of skills to form a broader base of competencies for poverty reduction. The success of the pilot led the Ministry of Agriculture and Forestry to ask how it could be scaled up to serve a larger proportion of the 6,000 extension workers throughout the country. But the unavailability of a sufficient number of qualified teachers and the fact that the poorest districts are also the most remote posed great challenges.

In 2011 the PRAM Knowledge Sharing Network (PRAM-KSN) was developed as a web-based platform to accelerate capacity building among extension workers [3]. The goal of PRAM-KSN was to facilitate peer-to-peer learning among agricultural extension workers. A key design element of the platform was allowing user-authored multimedia content to be uploaded and shared. Uptake of the system was rapid and as of February 2012 there were already active users in 18 districts over 8 southern provinces of Lao PDR.

One topic often addressed in PRAM-KSN was how to deal with reoccurring destructive events. For example, in severe weather conditions and disaster situations such as drought or flooding, people in rural and remote districts suffer from limited support. Hence, bi-directional and rapid communication channels between citizens and higher administrative officers was required to minimize disaster risk [6]. As a reaction to this, the project Mobile for Development (Mobile4D) [7] was established in 2012 within the activities of the Capacity Lab. About 15 students of the faculty of computer science at the University of Bremen worked in collaboration with MAF, the E-Government Centre in Laos, and the National University of Laos (NUOL) to develop the mobile disaster alerting and reporting system. To get a better understanding of the development context, the team adopted hybrid research methodologies including: interviews, questionnaires, prototype developments, field visits, and discussion workshops.

Mobile4D was planned as real-time location-based reporting and alerting system. Moreover, it is integrated with PRAM-KSN to allow dual functionality in a single system. In 2013, Mobile4D was tested in Luang Prabang in Northern Laos. During this test, field studies and questioners had been conducted to collect feedback and to identify further development challenges and requirements.

As a result of active collaboration, it was decided to carry out an information/training session of Mobile4D. At the beginning of 2015, the 3-day session was held in Attapeu province for district officers from six districts. The session

12 http://www . wetlandsalliance.org/

13 http://www.pramlaos.org/ 


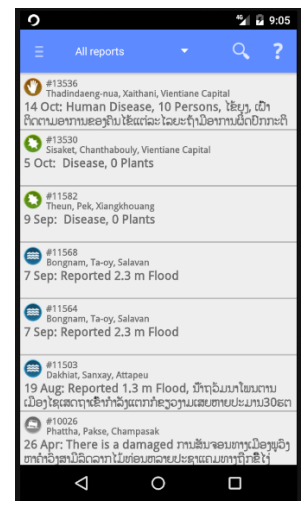

(a) report list

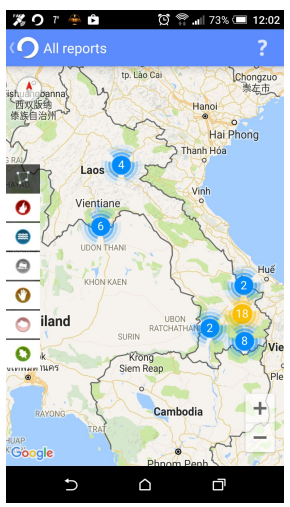

(b) report map

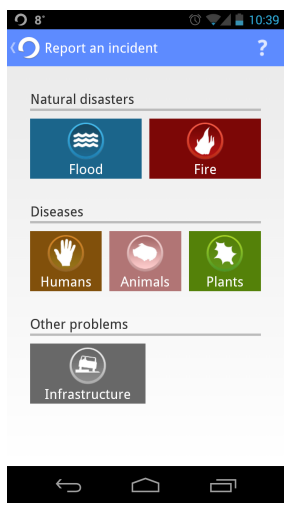

(c) disaster selector

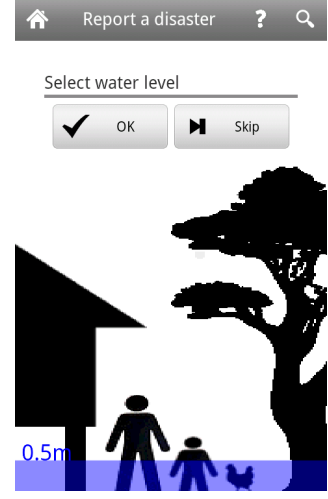

(d) flood level

Fig. 1: Impression of the mobile interface of the Mobile4D system

served as a pilot study of the system in 6 districts over three provinces: Phouvong and Xansay of Attapeu Province, Dakcheung and Kaleum in Xekong Province, and $\mathrm{Ta}$ 'Oy and Samuay of Salavan Province. The training was collaboratively conducted by computer scientists from the University of Bremen and trainers from MAF and NUOL. During training, participants were provided with the developed system on smart-phones. The training included deploying the system and becoming familiar with its functionality. After the training, each participant was able to utilize the developed system to send reports and to receive alerts. Moreover, each participant could act as a tutor for other district officers.

Since 2015, Mobile4D has been used in these three south Lao provinces. District officers and local staff use the system to report on small disasters and incidents that happened in their districts such as floods, infrastructure damage, and diseases. Afterward, MAF conducted an additional training session for district officers from eleven districts over five provinces, as well as participants from surrounded districts. Currently, we are investigating possible expansions of the system to the entire country of Laos aiming to achieve better reporting possibilities and to be able to provide better support where it is required. Figure 1 provides an impression of the interface of the mobile client of the system; Figure 1a shows a list of recently issued reports, while the map in Figure 1b demonstrates various filters of the reports based on type of disasters (flood, fire, ...etc.) or the status of reports (active/inactive). Whereas Figures 1c and 1d illustrate the easy-of-use requirements; Through interactive GUI users are able to interact with the system by easy ways.

\section{Use Cases of Mobile4D}

In this section, we introduce the primary use cases of the Mobile4D system and additional ones, which were identified during the pilot study in Laos. The 
use cases can be classified into three categories: reporting and alerting (Section 4.1), knowledge sharing (Section 4.2), and information distribution (Section 4.3). The first two scenarios (reporting and alerting and knowledge sharing) are the original scenarios of Mobile4D, while the last scenario is one of the upcoming extensions.

\subsection{Reporting and Alerting}

The main scenario during the development of the Mobile4D system was the crowdsourced disaster reporting and alerting. That is, if a disaster like flooding occurs, ordinary people act as sensors creating reports using a mobile phone. The reports are directly sent to all local people concerned as well as to all concerned authorities on all levels of administration. This reduces the reaction time as the information is directly available to everyone. And even more important, all other possibly affected individuals are informed and can immediately react, securing or rescuing livestock and tools.

At the moment, Mobile4D only addresses limited types of disasters: flooding, wildfires, animal disease, plant disease, human disease, and infrastructural damage. However, the pilot study in Laos showed that the possibility to add further types of disasters is required (e.g., drought or plagues like locusts).

\subsection{Knowledge Sharing}

Another use case of the Mobile4D system is the functionality of supporting direct information sharing between people. Specifically, to provide people in Laos with a possibility to communicate information regarding specific local problems. This resulted in the inclusion of information and material from the previously mentioned Poverty Reduction and Agricultural Management Knowledge Sharing Network (PRAM-KSN). Thus, the Mobile4D system allows its users to directly access especially the tutorials provided through the PRAM-KSN.

\subsection{Information Distribution}

A further result of the pilot study in Laos is the insight that people who do not use a system on a day-to-day basis may not be fully prepared to use it when they need to use it. To address this issue the additional scenario of distributing useful information on a daily basis was requested. This new extension aims to build trustworthiness between users and the proposed system.

One example of such daily information distribution are so-called farm gate prices. These are the prices a farmer can sell his goods for. However, farmers do not always know the day-to-day prices and it is difficult for them to communicate with other farmers in the area to determine the best current offer from companies. The idea is to extend Mobile4D to allow buyers to announce their offers through the system and provide this information directly to local farmers. This would allow farmers to easily determine where and to whom to sell their goods. 


\section{An Overview of the Mobile4D System}

The Mobile4D framework consists of three systems: a disaster server (Section 4.1), a web service (Section 4.2), and a mobile client (Section 4.3) [7]. In this section, we address the technologies used to implement the Mobile4D system and its functionality as it is currently deployed in Laos.

\subsection{The Disaster Server}

The disaster server is one of the two key pieces of the Mobile4D system (the other one is the mobile client). It allows to centrally collect information regarding disasters provided by individuals observing them. In addition, the server allows to access this information and it proactively informs authorities and individuals that either requested this information or will be directly affected by the event. The software is implemented in Java ${ }^{14}$, using the JPA/eclipselink/ $/ 2^{15}$ libraries to provide a database, and mosquitto ${ }^{16}$ as a push service.

\subsection{The Web Service}

The web service provides the possibility to access the disaster server and information provided by it through the Internet. Thus, this web service allows to report incidents or inspect reports. It is specifically designed to allow government authorities to receive and manage warnings and reports about current disaster events, and to send warnings and information material to affected people. It is implemented using HTML $5^{17}$ and JavaScript ${ }^{18}$.

\subsection{The Android Client}

The mobile client is an application that allows to provide and receive information regarding disaster events from almost anywhere. It is implemented as an application for Android ${ }^{19}$ smart phones. A main focus during the development was on the graphical user interface, in order to ensure a simple usage, i.e., intuitive and text-free. Figure 1c shows the first page of the disaster report creation process. Figure 1d shows the first approach to a text-free interface for the selection of the water level of a flooding incident. This interface allows the use of a touchscreen and provides an intuitive design to slide the water level to the observed height.

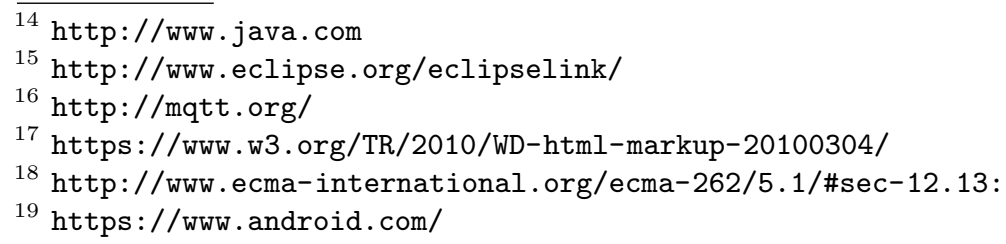




\subsection{The Mobile4D Framework}

The Mobile4D framework combines all three systems to create an integrated mobile crowdsourcing-based disaster reporting and alerting system. The disaster server provides an $\mathrm{REST}^{20}$ interface to manage information and supports $\mathrm{JSON}^{21}$ and Common Alerting Protocol $(\mathrm{CAP})^{22}$ to formalize disaster events. The REST interface is used by the mobile client and the web service to create and retrieve disaster reports on or from the server. In addition, the server uses a push service to directly inform affected people and government authorities by sending reports and information to their respective mobile clients.

\section{Experience with the System}

This section summaries the learned lessons and describes our experience with the Mobile4D. During a field visit in February 2016 we interviewed end users of the Mobile4D system in three districts in three separate provinces of southern Laos. The interviews were conducted with various enduser groups: district and province officers. These users utilize the system to report incidents on provincial and district levels. The most common reports concerned infrastructure damage such as landslide blockage of a new road in Dakcheung district. Other use was not anticipated in the original design of Mobile4D. This included new types of incidents as well as new ways of making use of the reports. An example of the former was the reporting of locusts in northern Laos. An example of the latter was the use of foot and mouth disease reports to determine locations to which vaccine needed to be distributed.

We learned that sometimes other reporting channels (e.g., email) still are used, that do not have the communicative advantages of Mobile4D; therefore it will be important to further encourage users to make their reports through Mobile4D.

Typically incidence reports are issued on a monthly basis on the district level, but only in cases of major incidents. However, it appears desirable to also record minor incidents in order to get the full picture and to keep the users fully familiar with the use of the system. Therefore it seems sensible to encourage users of Mobile4D to use the system more regularly, also for minor incidents and immediately when they occur. A way to make it more common for end users to work with the system could be to support users to also report positive events over the system to make Mobile4D a continuously used communication channel.

Currently reports made on the district level must be approved on the provincial level. In order to get urgent information as quickly as possible to directly affected community members, it should be considered to warn these citizens directly, but with not yet approved information marked as preliminary.

${ }^{20}$ http://www.ics.uci.edu/ fielding/pubs/dissertation/rest_arch_style.htm

${ }^{21}$ http://www.json.org/

${ }^{22}$ https://docs.oasis-open.org/emergency/cap/v1.2/CAP-v1.2-os.html 


\section{Conclusion and Future Work}

Experience with the pilot use of Mobile4D has resulted in identifying a range of desired new functionality. Users expressed a desire to have ready access to information concerning human and animal diseases. This could be retrieved using the information in the reports in order to suggest useful relevant information concerning a problem at hand. Reports of animal disease could also be supplemented by display of a buffer region for vaccine distribution purposes. This could be readily inferred from a map which can supply information about connectivity. Supplementary information would also be valuable for locust reporting, such as, wind speed and direction, which are highly relevant to spread of locusts. It would be useful to supplement each report with this information, which can be retrieved automatically from online services.

The pilot also raised implementation aspects that could be improved. For example, the feature to link photos to reports requires high bandwidth, which might be problematic in many parts of Laos. Therefore, a compression facility needs to be added to make it more usable. Finally, if Mobile4D can be linked to social media (e.g., Facebook) that could increase the day-to-day use of the system as well as the familiarity of the system among local people. In addition to new functionality, remote and personal training of users will be one of our priorities. The pilot study showed that this is absolutely essential for the success of the system.

We will also investigate possibilities to apply mobile crowdsourcing and artificial intelligence techniques (e.g., spatial reasoning and machine learning) to tackle issues related to vector-borne diseases like malaria and dengue. The idea is to generate and provide a prediction of the expected spatial and temporal pattern of transmission of the disease and its vector. The hypothesis is that the availability of this information will allow more timely and targeted intervention.

Last September 2016, we conducted a discussion workshop at the Capacity Lab with our partners. During this workshop, we discussed possible integration between Mobile4D and other services provided by the Lao E-government center. Furthermore, potential utilization of Mobile4D in other countries has been addressed.

\section{Acknowledgements}

We gratefully acknowledge valuable suggestions by Lutz Frommberger and Falko Schmid in numerous discussions.

This work was partially funded by the German Research Foundation (DFG) through the projects SOCIAL (FR 806/15) and Human-centered relational feature classification for VGI (FR 806/17) in the priority program VGIScience, as well as a Fellowship from the Hanse-Wissenschaftskolleg Institute for Advanced Study to Peter Haddawy. Moreover, we thank Lao DECIDE info phase III (CDE/SDC) and Food Nutrition Marketing Linkage (FNML/IFAD) for their support in the deployment of the test system and organizing training in Laos. 


\section{References}

1. Ali, M., Bailur, S.: The challenge of "sustainability" in ICT4D? Is bricolage the answer. In: Proceedings of the 9th international conference on social implications of computers in developing countries (2007)

2. Careem, M., De Silva, C., De Silva, R., Raschid, L., Weerawarana, S.: Sahana: Overview of a disaster management system. In: 2006 International Conference on Information and Automation. pp. 361-366. IEEE (2006)

3. Chew, H.E., Sort, B., Haddawy, P.: Building a crowdsourcing community: how online social learning helps in poverty reduction. In: Proceedings of the 3rd ACM Symposium on Computing for Development. p. 21. ACM (2013)

4. Déglise, C., Suggs, L.S., Odermatt, P.: SMS for disease control in developing countries: a systematic review of mobile health applications. Journal of Telemedicine and Telecare 18(5), 273-281 (2012)

5. Degrossi, L.C., de Albuquerque, J.P., Fava, M.C., Mendiondo, E.M.: Flood Citizen Observatory: a crowdsourcing-based approach for flood risk management in Brazil. In: SEKE. pp. 570-575 (2014)

6. Frommberger, L., Schmid, F.: Crowdsourced bi-directional disaster reporting and alerting on smartphones in Lao PDR. CoRR abs/1312.6036 (2013)

7. Frommberger, L., Schmid, F.: Mobile4d: crowdsourced disaster alerting and reporting. In: Proceedings of the Sixth International Conference on Information and Communications Technologies and Development: Notes-Volume 2. pp. 29-32. ACM (2013)

8. Gao, H., Barbier, G., Goolsby, R.: Harnessing the crowdsourcing power of social media for disaster relief. IEEE Intelligent Systems 26(3), 10-14 (2011)

9. Goodchild, M.F.: Citizens as sensors: the world of volunteered geography. GeoJournal 69(4), 211-221 (2007)

10. Goodchild, M.F., Glennon, J.A.: Crowdsourcing geographic information for disaster response: a research frontier. International Journal of Digital Earth 3(3), 231-241 (2010)

11. Haddawy, P., Sayakoummane, S.: ICT for poverty reduction in Lao PDR. UN Chronicle: The Digital Dividend 48(4) (2011)

12. Heeks, R.: ICT4D 2.0: The next phase of applying ICT for international development. Computer 41(6) (2008)

13. Kleine, D., Unwin, T.: Technological Revolution, Evolution and New Dependencies: what's new about ICT4D? Third World Quarterly 30(5), 1045-1067 (2009)

14. Mahmud, I., Akter, J., Rawshon, S.: SMS based disaster alert system in developing countries: A usability analysis. Internation Journal of Multidisciplinary Management Studies 2(4) (2012)

15. Muralidharan, S., Rasmussen, L., Patterson, D., Shin, J.H.: Hope for Haiti: An analysis of Facebook and Twitter usage during the earthquake relief efforts. Public Relations Review 37(2), 175-177 (2011)

16. Nawi, H.S.A., Rahman, A.A., Ibrahim, O., et al.: Government ICT project failure factors: project stakeholders' views. Journal of information systems research and innovation 2(1), 69-77 (2012)

17. Oh, O., Kwon, K., Rao, H.: An exploration of social media in extreme events: Rumor theory and twitter during the haiti earthquake 2010. In: ICIS 2010 Proceedings - Thirty First International Conference on Information Systems (2010)

18. Okolloh, O.: Ushahidi, or 'testimony': Web 2.0 tools for crowdsourcing crisis information. Participatory learning and action 59(1), 65-70 (2009) 
19. Palen, L., Liu, S.B.: Citizen communications in crisis: Anticipating a future of ict-supported public participation. In: Proceedings of the SIGCHI Conference on Human Factors in Computing Systems. pp. 727-736. CHI '07, ACM, New York, NY, USA (2007) 